\title{
Mechanical properties of Indonesian hyperelastic low-grade rubber for low-cost base isolator
}

\author{
Usman Wijaya ${ }^{1}$, Roesdiman Soegiarso ${ }^{1}$, and Tavio $^{2, *}$ \\ ${ }^{1}$ Department of Civil Engineering, Universitas Tarumanagara, Jakarta, Indonesia \\ ${ }^{2}$ Department of Civil Engineering, Institut Teknologi Sepuluh Nopember (ITS), Surabaya, Indonesia
}

\begin{abstract}
Most parts of Indonesia are located at high-risk seismic regions. Therefore, it is necessary to seriously consider earthquake in designing the seismic-resistant buildings. One of the most effective methods to reduce the impact of the earthquake force is to introduce the use of the elastomeric rubber base isolation as an effective earthquake damper. Indonesia as a tropical country has many sources of raw materials particularly rubber. This can be potential as a main basic need for producing an optimum elastomeric rubber base isolation system. This paper determined an approach method of constitutive low-grade rubber to get the best fitting with experimental data. Five deformation models such as Mooney-Rivlin, Ogden, Arruda-Boyce, Neo-Hookean, Yeoh models compare to uniaxial test were adopted to simulate the characteristics of rubber materials used in base isolation. From the analysis, it can be concluded that Yeoh model give the best fitting and the most accurate to the test data even in limited test data.
\end{abstract}

\section{Introduction}

One of the biggest rubber producers in the world is Indonesia. Indonesian rubber is widely used for industries such as automotive, aerospace industry, mechanical, and civil engineering. In the field of civil engineering rubber is used as an earthquake-resisting base isolation [1,2]. Rubber that is used for this paper is the category of waste rubber in the automotive industry and low-strain category. The use of base isolation in multi-storey buildings is evidenced to provide good performance in reducing damage caused by earthquake load [3,4]. Elastomeric base isolation used for damping earthquake loads consist of perforated steel sheet or fiber glass and vulcanized rubber under high temperatures - to form composite rubber bearings. However, its application for public low-cost housing has not been developed and implemented yet though several studies and researches have been begun $[5,6]$. Rubber has hyperelastic non-linear stress-strain characteristic, so it is suitable to be used as an earthquake damper base isolator $[7,8]$. Rubber has a large deformation and strain value up to $400 \%$.

\footnotetext{
*Corresponding author: tavio w@yahoo.com
} 
In general, the rubber's stress-strain stretching can attain from 500 to 1000 percent. The tensile stress-strain curve of rubber is non-linear hence Hooke's law cannot be used $[9,10]$.

To define the mathematical model of finite element analysis, for hyperelastic rubber, it is very important to do the experimental laboratory test such as uniaxial tension, planar shear test, etc. A planar shear test seems like a uniaxial tension test; however, the specimen is determined that the width of the specimen is considerably higher than thickness of the specimen. The importance of planar test configuration is that the substantial constrains aspect ratio protect thinning in width direction and producing in a plane stress-strain state. When perform a hyperelastic materials uniaxial tests are the simplest method to consider low strain only and it represented planar shear test.

The stress-strain experimental laboratory test created by the elastomer extension in some deformation method is required and "fitted" to simulate the rubber's mechanical properties. The experimental laboratory tests were carried out for curve fitting purpose.

\section{Constitutive model}

Consequently, the hyperelastic rubber material is anisotropic incompressible non-linear material can be illustrated as follows [11]:

$$
W=\sum_{i+j=1}^{N} C_{i j}\left(I_{1}-3\right)^{i}\left(I_{2}-3\right)^{j}
$$

where $C$ is a constant (example: $C_{0}, C_{1}$, etc.); $I$ value is a shear behavior of the hyperelastic behavior. To solve Eq. (1), it can be used Mooney-Rivlin and Neo Hookean models.

The hyperelastic constitutive has a derivation formula of stress-strain energy function $(W)$ from three invariant green deformation tensors as follows [11]:

$$
W=f\left(I_{1}, I_{2}, I_{3}\right)
$$

where:

$$
\begin{aligned}
& I_{1}=\lambda_{1}^{2}+\lambda_{2}^{2}+\lambda_{3}^{2} \\
& I_{2}=\lambda_{1}^{2} \lambda_{2}^{2}+\lambda_{2}^{2} \lambda_{3}^{2}+\lambda_{3}^{2} \lambda_{1}^{2} \\
& I_{3}=\lambda_{1}^{2}+\lambda_{2}^{2}+\lambda_{3}^{2}
\end{aligned}
$$

To determine elastic rubber-like material a hyperelastic model comprises of five deformation models such as Mooney-Rivlin, Ogden, Arruda-Boyce, Neo-Hookean, Yeoh is modeled.

\subsection{Mooney-rivlin model}

Mooney-Rivlin model can complete polynomial models for expansive strain in elongation and shear deformation essentially. The strain energy function of the Mooney-Rivlin model can be resolved as follows $[12,13]$ :

$$
W=C_{10}\left(\overline{I_{1}}-3\right)+C_{01}\left(\overline{I_{2}}-3\right)+\frac{1}{D_{1}}\left(J_{e l}-1\right)^{2}
$$


where $C_{10}=\frac{\mu_{0}}{2}$ is an initial shear modulus and $D$ is a constant.

\subsection{Neo hookean model}

Neo Hookean model is simpler than Mooney-Rivlin model formula, where in Neo Hookean model, $\mathrm{C}_{01}=0$. So, the form becomes as follows [15]:

$$
W=C_{10}\left(\overline{I_{1}}-3\right)
$$

\subsection{Ogden model}

Stress-strain curve of Ogden model is computed based on strain energy function of stretch ratio and derived form of two strain invariant $I_{1}$ and $I_{2}$. The model can be illustrated as follows [16]:

$$
W=\sum_{i=1}^{N} \frac{2 \mu_{i}}{a_{i}^{2}}\left(\lambda_{1}^{a_{i}}+\lambda_{2}^{a_{i}}+\lambda_{3}^{a_{i}}-3\right)+\sum_{i=1}^{N} \frac{1}{D_{i}}\left(J_{e l}-1\right)^{2 i}
$$

where: $\lambda_{1}^{2}+\lambda_{2}^{2}+\lambda_{3}^{2}=1 ; I_{1}$ and $I_{2}$ is strain energy function of Ogden model.

\subsection{Arruda-boyce model}

Strain energy function of Arruda-Boyce model is determined as follows [16]:

$$
W=\mu_{0} \sum_{i=1}^{5} \frac{C_{i}}{\lambda_{m}^{2 i-2}}\left(I_{1}^{i}-3\right)+\frac{1}{D}\left[\frac{J_{e l}^{2}-1}{2}-\ln \left(J_{e l}\right)\right]
$$

where $\mathrm{C}$ is a constant and $\lambda_{\mathrm{m}}$ is locking stretch ratio.

\subsection{Yeoh model}

Another name of Yeoh model is reduce polynomial model. Strain energy function of Yeoh model is higher order of Neo Hookean model, where It obtained as follows [17, 18]:

$$
W=\sum_{i=1}^{3} C_{10}\left(\bar{I}_{1}-3\right)^{i}+\sum_{i=1}^{3} \frac{1}{D_{i}}\left(J_{e l}-1\right)^{2 i}
$$

\section{Experimental tests}

Testing standard based on ISO 37:2011 [19] were conducted. In hyperelastic material testing, uniaxial tension behavior is similar to biaxial compression test, then biaxial tension test equals to uniaxial compression test and planar test both tension and compression is the same. Volumetric test is not necessary because hyperelastic material is an incompressibility material. So that, for low strain consideration hyperelastic experimental test only uniaxial tension represents others. 


\subsection{Hardness IRHD test}

The hardness tests in the range of 53 to 55 shore A durometer scale in the IRHD (International Rubber Hardness Degrees) in the soft rubber categories mention as L1, L2 and $\mathrm{L} 3$ respectively.

\subsection{Uniaxial Tensile Test}

The test's basic necessity is to serve a condition of natural strain to make the specimen to have longer extension in the smaller width than its width and thickness's extensions. The rubber test specimens were dogbone based on ISO 37:2011 [19] with the following sizes shown in Figure 1. The test transformed into accomplished at the dogbone-formed specimens with overall length of $115 \mathrm{~mm}$, flange of $25 \mathrm{~mm}$ width, narrow part of $33 \mathrm{~mm}$ length, narrow part of $6.2 \mathrm{~mm}$ width, $14 \mathrm{~mm}$ outside transition radius, and $25 \mathrm{~mm}$ inside transition radius.

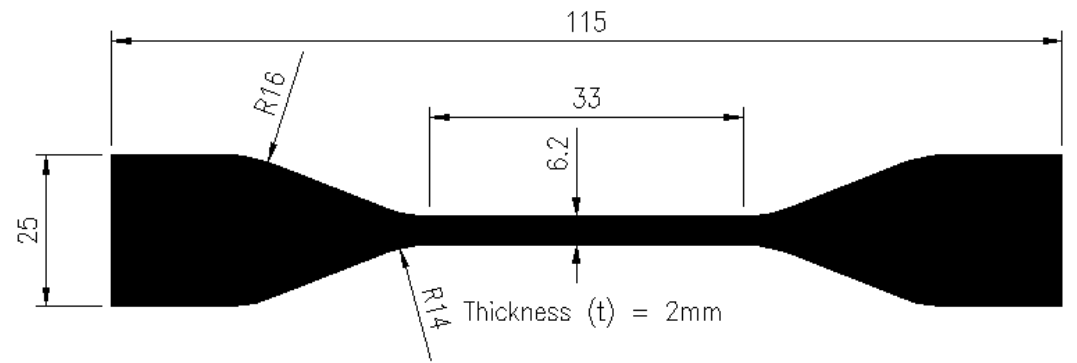

Fig. 1. Uniaxial tensile test specimen dimension.

In uniaxial tension hyperelastic material, stretch ratio is obtained as follows [20]:

$$
\lambda_{1}=\lambda ; \lambda_{2}=\lambda_{3}=\frac{1}{\sqrt{\lambda}}
$$

and stress of uniaxial tension is obtained as follows:

$$
\sigma_{1}=\sigma=\lambda \frac{\partial W(\lambda)}{\partial \lambda}, \sigma_{2}=\sigma_{3}=0
$$

where $W(\lambda)=W\left(\lambda, \lambda^{-0.5}, \lambda^{0.5}\right)$ is a strain energy density or function.

\section{Curve fitting and experimental results}

Every constitutive model is computed to get a suitable curve fitting low-grade rubber constant. The coefficient that was met the constitutive model can be found in Table 1 . Strain energy function in hyperelastic rubber uniaxial test model, equibiaxial test and planar test compared with experimental test data, it acquired and identified hyperelasticity material fitting the test. The test data are specified as nominal stress-nominal strain data pairs for uniaxial, planar, and biaxial tests to determine the shear constants Cij that can control the shear behavior. Generates an equation for the stress in terms of the strain invariants or stretches and the unknown hyperelastic constants. Overall from five constitutive model 
Table 1. Coefficient of constitutive model.

\begin{tabular}{|c|c|c|c|}
\hline \multicolumn{2}{|c|}{ Model } & Constant & Unit \\
\hline \multirow{4}{*}{ Arruda-Boyce } & $\mu$ & 0.383854 & $\mathrm{MPa}$ \\
\cline { 2 - 4 } & $\mu_{0}$ & 0.383854 & $\mathrm{MPa}$ \\
\cline { 2 - 4 } & $\lambda_{\mathrm{m}}$ & 2369.145 & $\mathrm{MPa}$ \\
\hline \multirow{4}{*}{ Yeoh } & $C_{10}$ & 0.201947 & $\mathrm{MPa}$ \\
\cline { 2 - 4 } & $C_{20}$ & $-4.0756 \mathrm{e}-03$ & $\mathrm{MPa}$ \\
\cline { 2 - 4 } & $C_{30}$ & $3.3320 \mathrm{e}-04$ & $\mathrm{MPa}$ \\
\hline \multirow{3}{*}{ Ogden } & $\mu_{1}$ & 3.82329 & $\mathrm{MPa}$ \\
\cline { 2 - 4 } & $\alpha_{1}$ & 4.25347 & $\mathrm{MPa}$ \\
\hline \multirow{3}{*}{ Mooney-Rivlin } & $C_{10}$ & 0.15877 & $\mathrm{MPa}$ \\
\cline { 2 - 4 } & $C_{01}$ & $2.9437 \mathrm{e}-02$ & $\mathrm{MPa}$ \\
\hline \multirow{2}{*}{ Neo Hookean } & $C_{10}$ & 0.19192 & $\mathrm{MPa}$ \\
\hline
\end{tabular}

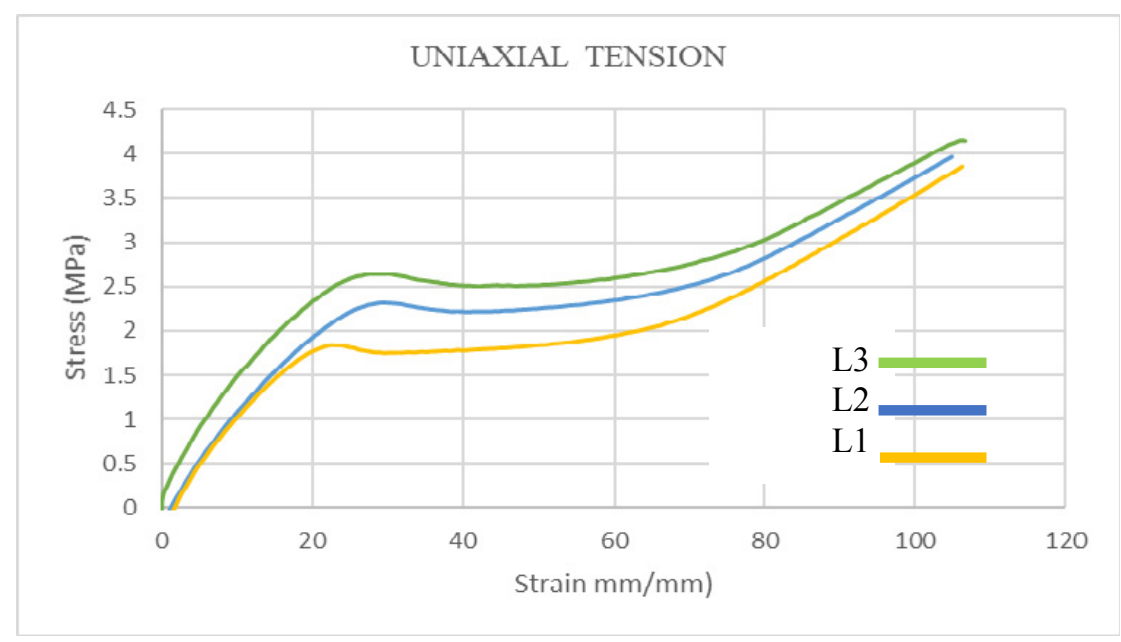

Fig. 2. Uniaxial tensile test.

shown in Table 1, The Yeoh model and Arruda-Boyce give the complete constant fitting even in limited test data, for example, It only has simple tension data or uniaxial data.

From Figure 2, the results of the rubber test show that the lowest hardness produces the lowest stress, a linear increase in hardness with stresses. Three samples compared to the constitutive model, it is still close to the results of the constitutive model, which means that the uniaxial test is sufficient to be used to determine the characteristics of the low strain rubber properties. The three types of rubber break at strain below 200 percent since the base isolator used is indeed a low-cost category so that when used for small axial loads it is still adequate. 


\section{Conclusions}

In this paper, experimental data from five test deformations were obtained to align the hyperelastic model constant for low-grade rubber behavior. Measure strain energy function of the uniaxial, planar, and biaxial specimen. The Yeoh model appeared to be the most suitable choice for predicting the hysteretic constant of the low-grade rubber composition in simple tension as a result of its capacity to match experimental data points at small and large strain values under $200 \%$ strain. Yeoh model gives the best fitting and the most accurate to the test data even, and it just has primary tension data or uniaxial data Yeoh model provides a stable analytical description of the stress-strain response material.

The authors would like to gratefully acknowledge for all the facilities and the supports received to make this research possible.

\section{References}

1. A.B. Habieb, G. Milani, Tavio, F. Milani, Open Civil Eng. J. 11, M2 (2017)

2. Tavio, H. Sugihardjo, A. Purniawan, Y. Lesmana, AIP Conference Proc. 1903 (2017)

3. A.B. Habieb, G. Milani, Tavio, Eng. Failure Analysis 90 (2018)

4. H. Sugihardjo, Tavio, Y. Lesmana, Int. J. of Appl. Eng. Res. 11, 14 (2016)

5. A.B. Habieb, G. Milani, Tavio, F. Milani, AIP Conference Proc. 1906 (2017)

6. H. Sugihardjo, Tavio, Y. Lesmana, Civil Eng. J. 4, 1( 2018)

7. A.B. Habieb, G. Milani, Tavio, F. Milani, AIP Conference Proc. 1863 (2017)

8. A.B. Habieb, G. Milani, Tavio, F. Milani, WIT Trans. on the Built Env. 172 (2017)

9. Z. Guo, L.J. Sluys, Int. J. of Solids and Struct. 43 (2006)

10. B.T.W. Wijaya, Tavio, Int. J. of Civil Eng. Tech. 10 (2019)

11. M. Shahzad, A. Kamran, M.Z. Siddiqui, M. Farhan, Materials Res. 18, 5 (2015)

12. M. Mooney. J. of Appl. Phys. 11, 9 (1940)

13. R.S. Rivlin. Philosophical Trans. of the Royal Soc. 241, 835 (1948)

14. B. Kim, B.S. Lee, S.J. Cho, H. Park, S. Yeom, H.S. Park, Int. J. of Precision Eng. and Manufacturing 13, 5 (2012)

15. R.W. Ogden, R. Hill, Proc. of the Royal Soc. London A 3261567 (1927)

16. E.M. Arruda, M.C. Boyce, J. of the Mech. and Phys. of Solids 41, 2 (1993)

17. O.H. Yeoh, Rubber Chem. and Tech. 66, 5 (1993)

18. A.N. Gent, Rubber Chem. and Tech. 69, 1 (1996)

19. International Standard Organization, Rubber vulcanized Rubber vulcanized or thermoplastic-determination of tensile stress-strain properties ISO 37: 2011 (International Standard Organization, Geneva, 2011)

20. R.W. Ogden, G. Saccomandi, I. Sgura, Computational Mech. 34, 6 (2004) 